Supplement of Biogeosciences, 15, 6417-6437, 2018

https://doi.org/10.5194/bg-15-6417-2018-supplement

(C) Author(s) 2018. This work is distributed under

the Creative Commons Attribution 4.0 License.

(c) (1)

Supplement of

\title{
High variability of particulate organic carbon export along the North Atlantic GEOTRACES section GA01 as deduced from ${ }^{234} \mathrm{Th}$ fluxes
}

Nolwenn Lemaitre et al.

Correspondence to: Nolwenn Lemaitre (nolwenn.lemaitre@erdw.ethz.ch)

The copyright of individual parts of the supplement might differ from the CC BY 4.0 License. 
Table S1: ${ }^{238} \mathrm{U}$ and total ${ }^{234} \mathrm{Th}$ activities in $\mathrm{dpm} \mathrm{L}^{-1}$ and resulting ${ }^{234} \mathrm{Th} /{ }^{238} \mathrm{U}$ ratios ${ }^{238} \mathrm{U}$ activity is derived from salinity (see text).

\begin{tabular}{|c|c|c|c|c|c|c|c|c|c|c|}
\hline \multirow[t]{2}{*}{$\begin{array}{c}\text { Station } \\
\#\end{array}$} & \multirow{2}{*}{$\begin{array}{c}\text { Depth } \\
\text { m } \\
19\end{array}$} & \multirow{2}{*}{$\begin{array}{c}\begin{array}{c}\text { Temperature } \\
{ }^{\circ} \mathrm{C}\end{array} \\
15.89\end{array}$} & \multirow{2}{*}{$\begin{array}{c}\begin{array}{c}\text { Salinity } \\
\text { psu }\end{array} \\
35.27\end{array}$} & \multicolumn{3}{|c|}{$\begin{array}{c}{ }^{238} U \\
\operatorname{dpm} \cdot L^{-1}\end{array}$} & \multicolumn{3}{|c|}{$\begin{array}{c}{ }^{234} \mathrm{Th} \\
\mathrm{dpm} \cdot \mathrm{L}^{-1}\end{array}$} & \multirow{2}{*}{$\begin{array}{c}{ }^{234} \mathrm{Th} /{ }^{238} \mathrm{U} \\
0.74\end{array}$} \\
\hline & & & & 2.46 & \pm & 0.05 & 1.83 & \pm & 0.06 & \\
\hline \multirow{16}{*}{$\begin{array}{c}\# 1 \\
40.3^{\circ} \mathrm{N} /-10.0^{\circ} \mathrm{E} \\
20 / 05 / 2014\end{array}$} & 39 & 14.93 & 35.55 & 2.48 & \pm & 0.05 & 1.69 & \pm & 0.05 & 0.68 \\
\hline & 60 & 13.81 & 35.68 & 2.49 & \pm & 0.05 & 2.11 & \pm & 0.07 & 0.85 \\
\hline & 79 & 13.44 & 35.71 & 2.49 & \pm & 0.05 & 2.39 & \pm & 0.07 & 0.96 \\
\hline & 98 & 13.21 & 35.74 & 2.49 & \pm & 0.05 & 2.55 & \pm & 0.08 & 1.02 \\
\hline & 119 & 13.02 & 35.73 & 2.49 & \pm & 0.05 & 2.50 & \pm & 0.08 & 1.00 \\
\hline & 137 & 12.88 & 35.73 & 2.49 & \pm & 0.05 & 2.34 & \pm & 0.07 & 0.94 \\
\hline & 158 & 12.73 & 35.71 & 2.49 & \pm & 0.05 & 2.44 & \pm & 0.08 & 0.98 \\
\hline & 199 & 12.55 & 35.71 & 2.49 & \pm & 0.05 & 2.54 & \pm & 0.08 & 1.02 \\
\hline & 246 & 12.29 & 35.69 & 2.49 & \pm & 0.05 & 2.60 & \pm & 0.08 & 1.05 \\
\hline & 298 & 12.08 & 35.68 & 2.49 & \pm & 0.05 & 2.67 & \pm & 0.08 & 1.07 \\
\hline & 396 & 11.69 & 35.65 & 2.49 & \pm & 0.05 & 2.59 & \pm & 0.08 & 1.04 \\
\hline & 496 & 11.43 & 35.67 & 2.49 & \pm & 0.05 & 2.43 & \pm & 0.07 & 0.98 \\
\hline & 589 & 11.41 & 35.77 & 2.50 & \pm & 0.05 & 2.61 & \pm & 0.08 & 1.05 \\
\hline & 695 & 11.60 & 35.94 & 2.51 & \pm & 0.05 & 2.58 & \pm & 0.08 & 1.03 \\
\hline & 791 & 11.72 & 36.07 & 2.52 & \pm & 0.05 & 2.90 & \pm & 0.09 & 1.15 \\
\hline & 989 & 11.34 & 36.15 & 2.53 & \pm & 0.05 & 2.68 & \pm & 0.08 & 1.06 \\
\hline \multirow{18}{*}{$\begin{array}{c}\# 13 \\
41.4^{\circ} \mathrm{N} /-13.9^{\circ} \mathrm{E} \\
25 / 05 / 2014\end{array}$} & 20 & 15.49 & 35.85 & 2.50 & \pm & 0.05 & 1.75 & \pm & 0.05 & 0.70 \\
\hline & 40 & 14.86 & 35.81 & 2.50 & \pm & 0.05 & 1.83 & \pm & 0.05 & 0.73 \\
\hline & 60 & 13.30 & 35.76 & 2.50 & \pm & 0.05 & 2.23 & \pm & 0.07 & 0.90 \\
\hline & 78 & 13.14 & 35.76 & 2.50 & \pm & 0.05 & 2.31 & \pm & 0.06 & 0.93 \\
\hline & 99 & 12.99 & 35.75 & 2.49 & \pm & 0.05 & 2.26 & \pm & 0.07 & 0.91 \\
\hline & 119 & 12.93 & 35.75 & 2.49 & \pm & 0.05 & 2.63 & \pm & 0.08 & 1.05 \\
\hline & 139 & 12.88 & 35.75 & 2.49 & \pm & 0.05 & 2.82 & \pm & 0.07 & 1.13 \\
\hline & 159 & 12.83 & 35.74 & 2.49 & \pm & 0.05 & 2.64 & \pm & 0.07 & 1.06 \\
\hline & 197 & 12.64 & 35.71 & 2.49 & \pm & 0.05 & 2.52 & \pm & 0.07 & 1.01 \\
\hline & 248 & 12.38 & 35.68 & 2.49 & \pm & 0.05 & 2.65 & \pm & 0.07 & 1.06 \\
\hline & 298 & 12.20 & 35.66 & 2.49 & \pm & 0.05 & 2.50 & \pm & 0.07 & 1.00 \\
\hline & 398 & 11.82 & 35.62 & 2.48 & \pm & 0.05 & 2.52 & \pm & 0.07 & 1.01 \\
\hline & 496 & 11.59 & 35.62 & 2.48 & \pm & 0.05 & 2.66 & \pm & 0.08 & 1.07 \\
\hline & 594 & 11.23 & 35.61 & 2.48 & \pm & 0.05 & 2.61 & \pm & 0.07 & 1.05 \\
\hline & 693 & 10.66 & 35.57 & 2.48 & \pm & 0.05 & 2.55 & \pm & 0.08 & 1.03 \\
\hline & 792 & 10.55 & 35.68 & 2.49 & \pm & 0.05 & 2.35 & \pm & 0.06 & 0.95 \\
\hline & 990 & 9.33 & 35.65 & 2.49 & \pm & 0.05 & 2.63 & \pm & 0.08 & 1.06 \\
\hline & 1485 & 6.73 & 35.45 & 2.47 & \pm & 0.05 & 2.48 & \pm & 0.07 & 1.00 \\
\hline
\end{tabular}




\begin{tabular}{|c|c|c|c|c|c|c|c|c|c|c|}
\hline \multirow[t]{2}{*}{$\begin{array}{c}\text { Station } \\
\#\end{array}$} & \multirow{2}{*}{$\begin{array}{c}\text { Depth } \\
\text { m } \\
10\end{array}$} & \multirow{2}{*}{$\begin{array}{c}\begin{array}{c}\text { Temperature } \\
{ }^{\circ} \mathrm{C}\end{array} \\
14.44\end{array}$} & \multirow{2}{*}{$\begin{array}{c}\begin{array}{c}\text { Salinity } \\
\text { psu }\end{array} \\
35.68\end{array}$} & \multicolumn{3}{|c|}{$\begin{array}{c}{ }^{238} U \\
\text { dpm. } .^{-1}\end{array}$} & \multicolumn{3}{|c|}{$\begin{array}{c}{ }^{234} \mathrm{Th} \\
\mathrm{dpm} \cdot \mathrm{L}^{-1}\end{array}$} & \multirow{2}{*}{$\begin{array}{c}{ }^{234} \mathrm{Th} /{ }^{238} \mathrm{U} \\
0.57\end{array}$} \\
\hline & & & & 2.49 & \pm & 0.05 & 1.41 & \pm & 0.05 & \\
\hline \multirow{16}{*}{$\begin{array}{c}\# 21 \\
46.5^{\circ} \mathrm{N} /-19.7^{\circ} \mathrm{E} \\
31 / 05 / 2014\end{array}$} & 20 & 13.65 & 35.66 & 2.49 & \pm & 0.05 & 1.69 & \pm & 0.05 & 0.68 \\
\hline & 39 & 12.79 & 35.64 & 2.49 & \pm & 0.05 & 1.79 & \pm & 0.05 & 0.72 \\
\hline & 59 & 12.71 & 35.65 & 2.49 & \pm & 0.05 & 1.80 & \pm & 0.05 & 0.72 \\
\hline & 76 & 12.63 & 35.67 & 2.49 & \pm & 0.05 & 2.07 & \pm & 0.06 & 0.83 \\
\hline & 100 & 12.45 & 35.69 & 2.49 & \pm & 0.05 & 2.36 & \pm & 0.07 & 0.95 \\
\hline & 119 & 12.36 & 35.68 & 2.49 & \pm & 0.05 & 2.62 & \pm & 0.07 & 1.05 \\
\hline & 158 & 12.23 & 35.66 & 2.49 & \pm & 0.05 & 2.53 & \pm & 0.07 & 1.02 \\
\hline & 198 & 12.08 & 35.64 & 2.49 & \pm & 0.05 & 2.70 & \pm & 0.07 & 1.09 \\
\hline & 296 & 11.80 & 35.60 & 2.48 & \pm & 0.05 & 2.83 & \pm & 0.07 & 1.14 \\
\hline & 396 & 11.38 & 35.55 & 2.48 & \pm & 0.05 & 2.61 & \pm & 0.07 & 1.05 \\
\hline & 495 & 11.01 & 35.50 & 2.48 & \pm & 0.05 & 2.65 & \pm & 0.08 & 1.07 \\
\hline & 594 & 10.74 & 35.47 & 2.47 & \pm & 0.05 & 2.52 & \pm & 0.07 & 1.02 \\
\hline & 693 & 9.78 & 35.32 & 2.46 & \pm & 0.05 & 2.56 & \pm & 0.07 & 1.04 \\
\hline & 792 & 8.77 & 35.29 & 2.46 & \pm & 0.05 & 2.69 & \pm & 0.08 & 1.09 \\
\hline & 989 & 7.47 & 35.32 & 2.46 & \pm & 0.05 & 2.58 & \pm & 0.07 & 1.05 \\
\hline & 1189 & 6.17 & 35.21 & 2.45 & \pm & 0.05 & 2.36 & \pm & 0.07 & 0.96 \\
\hline \multirow{9}{*}{$\begin{array}{c}\# 26 \\
50.3^{\circ} \mathrm{N} /-22.6^{\circ} \mathrm{E} \\
04 / 06 / 2014\end{array}$} & 20 & 11.69 & 35.34 & 2.46 & \pm & 0.05 & 1.90 & \pm & 0.06 & 0.77 \\
\hline & 49 & 9.77 & 35.19 & 2.45 & \pm & 0.05 & 1.90 & \pm & 0.06 & 0.77 \\
\hline & 74 & 9.65 & 35.19 & 2.45 & \pm & 0.05 & 1.96 & \pm & 0.06 & 0.80 \\
\hline & 99 & 9.40 & 35.16 & 2.45 & \pm & 0.05 & 2.07 & \pm & 0.07 & 0.85 \\
\hline & 199 & 8.88 & 35.17 & 2.45 & \pm & 0.05 & 2.46 & \pm & 0.08 & 1.00 \\
\hline & 297 & 8.17 & 35.07 & 2.44 & \pm & 0.05 & 2.36 & \pm & 0.07 & 0.97 \\
\hline & 397 & 7.18 & 34.95 & 2.43 & \pm & 0.05 & 2.47 & \pm & 0.08 & 1.01 \\
\hline & 593 & 5.97 & 35.00 & 2.44 & \pm & 0.05 & 2.59 & \pm & 0.08 & 1.07 \\
\hline & 792 & 5.23 & 35.03 & 2.44 & \pm & 0.05 & 2.60 & \pm & 0.08 & 1.07 \\
\hline \multirow{16}{*}{$\begin{array}{c}\# 32 \\
55.5^{\circ} \mathrm{N} /-26.7^{\circ} \mathrm{E} \\
08 / 06 / 2014\end{array}$} & 11 & 10.51 & 35.13 & 2.45 & \pm & 0.05 & 1.63 & \pm & 0.08 & 0.67 \\
\hline & 20 & 10.48 & 35.13 & 2.45 & \pm & 0.05 & 1.58 & \pm & 0.06 & 0.65 \\
\hline & 40 & 8.89 & 35.07 & 2.44 & \pm & 0.05 & 1.88 & \pm & 0.06 & 0.77 \\
\hline & 60 & 8.60 & 35.08 & 2.44 & \pm & 0.05 & 1.87 & \pm & 0.05 & 0.77 \\
\hline & 99 & 8.67 & 35.13 & 2.45 & \pm & 0.05 & 1.64 & \pm & 0.05 & 0.67 \\
\hline & 119 & 8.08 & 35.04 & 2.44 & \pm & 0.05 & 2.32 & \pm & 0.06 & 0.95 \\
\hline & 139 & 7.88 & 35.03 & 2.44 & \pm & 0.05 & 2.59 & \pm & 0.08 & 1.06 \\
\hline & 160 & 8.23 & 35.12 & 2.45 & \pm & 0.05 & 2.48 & \pm & 0.07 & 1.01 \\
\hline & 199 & 7.99 & 35.09 & 2.44 & \pm & 0.05 & 2.42 & \pm & 0.07 & 0.99 \\
\hline & 298 & 7.05 & 34.98 & 2.43 & \pm & 0.05 & 2.48 & \pm & 0.07 & 1.02 \\
\hline & 376 & 5.99 & 34.89 & 2.43 & \pm & 0.05 & 2.52 & \pm & 0.07 & 1.04 \\
\hline & 446 & 6.36 & 35.04 & 2.44 & \pm & 0.05 & 2.47 & \pm & 0.07 & 1.01 \\
\hline & 494 & 5.70 & 34.98 & 2.43 & \pm & 0.05 & 2.50 & \pm & 0.07 & 1.03 \\
\hline & 593 & 5.15 & 34.98 & 2.43 & \pm & 0.05 & 2.52 & \pm & 0.08 & 1.04 \\
\hline & 693 & 4.78 & 34.96 & 2.43 & \pm & 0.05 & 2.45 & \pm & 0.07 & 1.01 \\
\hline & 792 & 4.48 & 34.94 & 2.43 & \pm & 0.05 & 2.29 & \pm & 0.06 & 0.94 \\
\hline
\end{tabular}




\begin{tabular}{|c|c|c|c|c|c|c|c|c|c|c|}
\hline \multirow[t]{2}{*}{$\begin{array}{c}\text { Station } \\
\#\end{array}$} & \multirow{2}{*}{$\begin{array}{c}\text { Depth } \\
\mathrm{m} \\
10\end{array}$} & \multirow{2}{*}{$\begin{array}{c}\text { Temperature } \\
{ }^{\circ} \mathrm{C}\end{array}$} & \multirow{2}{*}{$\begin{array}{c}\text { Salinity } \\
\text { psu }\end{array}$} & \multicolumn{3}{|c|}{$\begin{array}{c}{ }^{238} U \\
\operatorname{dpm} \cdot L^{-1}\end{array}$} & \multicolumn{3}{|c|}{$\begin{array}{c}{ }^{234} \mathrm{Th} \\
\mathrm{dpm} \cdot \mathrm{L}^{-1}\end{array}$} & \multirow{2}{*}{$\frac{{ }^{234} \mathrm{Th} /{ }^{238} \mathrm{U}}{0.50}$} \\
\hline & & & & 2.44 & \pm & 0.05 & 1.23 & \pm & 0.04 & \\
\hline \multirow{16}{*}{$\begin{array}{c}\# 38 \\
58.8^{\circ} \mathrm{N} /-31.3^{\circ} \mathrm{E} \\
11 / 06 / 2014\end{array}$} & 19 & 9.18 & 35.06 & 2.44 & \pm & 0.05 & 1.48 & \pm & 0.05 & 0.61 \\
\hline & 39 & 8.22 & 35.08 & 2.44 & \pm & 0.05 & 2.12 & \pm & 0.07 & 0.87 \\
\hline & 60 & 8.00 & 35.11 & 2.44 & \pm & 0.05 & 2.38 & \pm & 0.07 & 0.97 \\
\hline & 78 & 7.73 & 35.11 & 2.44 & \pm & 0.05 & 2.45 & \pm & 0.07 & 1.00 \\
\hline & 99 & 7.73 & 35.13 & 2.45 & \pm & 0.05 & 2.61 & \pm & 0.08 & 1.07 \\
\hline & 118 & 7.68 & 35.14 & 2.45 & \pm & 0.05 & 2.61 & \pm & 0.08 & 1.07 \\
\hline & 138 & 7.67 & 35.14 & 2.45 & \pm & 0.05 & 2.41 & \pm & 0.07 & 0.99 \\
\hline & 158 & 7.62 & 35.14 & 2.45 & \pm & 0.05 & 2.43 & \pm & 0.07 & 0.99 \\
\hline & 178 & 7.59 & 35.14 & 2.45 & \pm & 0.05 & 2.51 & \pm & 0.07 & 1.02 \\
\hline & 198 & 7.60 & 35.15 & 2.45 & \pm & 0.05 & 2.46 & \pm & 0.07 & 1.01 \\
\hline & 298 & 7.41 & 35.14 & 2.45 & \pm & 0.05 & 2.40 & \pm & 0.08 & 0.98 \\
\hline & 396 & 7.21 & 35.13 & 2.45 & \pm & 0.05 & 2.50 & \pm & 0.09 & 1.02 \\
\hline & 494 & 6.86 & 35.11 & 2.44 & \pm & 0.05 & 2.45 & \pm & 0.09 & 1.00 \\
\hline & 593 & 6.32 & 35.09 & 2.44 & \pm & 0.05 & 2.48 & \pm & 0.08 & 1.01 \\
\hline & 693 & 5.68 & 35.05 & 2.44 & \pm & 0.05 & 2.54 & \pm & 0.09 & 1.04 \\
\hline & 791 & 5.00 & 35.01 & 2.44 & \pm & 0.05 & 2.51 & \pm & 0.09 & 1.03 \\
\hline \multirow{17}{*}{$\begin{array}{c}\# 44 \\
59.6^{\circ} \mathrm{N} /-38.9^{\circ} \mathrm{E} \\
14 / 06 / 2014\end{array}$} & 9 & 6.83 & 34.85 & 2.42 & \pm & 0.05 & 1.91 & \pm & 0.06 & 0.79 \\
\hline & 20 & 6.80 & 34.85 & 2.42 & \pm & 0.05 & 2.18 & \pm & 0.06 & 0.90 \\
\hline & 40 & 5.07 & 34.89 & 2.43 & \pm & 0.05 & 2.42 & \pm & 0.07 & 1.00 \\
\hline & 59 & 4.49 & 34.87 & 2.43 & \pm & 0.05 & 2.46 & \pm & 0.07 & 1.01 \\
\hline & 79 & 4.33 & 34.90 & 2.43 & \pm & 0.05 & 2.31 & \pm & 0.06 & 0.95 \\
\hline & 99 & 4.28 & 34.91 & 2.43 & \pm & 0.05 & 2.40 & \pm & 0.06 & 0.99 \\
\hline & 118 & 4.12 & 34.89 & 2.43 & \pm & 0.05 & 2.44 & \pm & 0.07 & 1.00 \\
\hline & 138 & 4.03 & 34.89 & 2.43 & \pm & 0.05 & 2.23 & \pm & 0.05 & 0.92 \\
\hline & 158 & 4.04 & 34.89 & 2.43 & \pm & 0.05 & 2.42 & \pm & 0.07 & 1.00 \\
\hline & 198 & 4.00 & 34.89 & 2.43 & \pm & 0.05 & 2.35 & \pm & 0.06 & 0.97 \\
\hline & 297 & 3.92 & 34.89 & 2.43 & \pm & 0.05 & 2.56 & \pm & 0.06 & 1.05 \\
\hline & 396 & 3.88 & 34.89 & 2.43 & \pm & 0.05 & 2.56 & \pm & 0.07 & 1.05 \\
\hline & 495 & 3.79 & 34.88 & 2.43 & \pm & 0.05 & 2.38 & \pm & 0.06 & 0.98 \\
\hline & 594 & 3.73 & 34.88 & 2.43 & \pm & 0.05 & 2.35 & \pm & 0.05 & 0.97 \\
\hline & 692 & 3.63 & 34.87 & 2.43 & \pm & 0.05 & 2.62 & \pm & 0.06 & 1.08 \\
\hline & 792 & 3.61 & 34.87 & 2.43 & \pm & 0.05 & 2.23 & \pm & 0.06 & 0.92 \\
\hline & 1087 & 3.70 & 34.89 & 2.43 & \pm & 0.05 & 2.43 & \pm & 0.07 & 1.00 \\
\hline
\end{tabular}




\begin{tabular}{|c|c|c|c|c|c|c|c|c|c|c|}
\hline \multirow[t]{2}{*}{$\begin{array}{c}\text { Station } \\
\# \\
\end{array}$} & \multirow{2}{*}{$\begin{array}{c}\text { Depth } \\
\mathrm{m} \\
11\end{array}$} & \multirow{2}{*}{$\begin{array}{c}\begin{array}{c}\text { Temperature } \\
{ }^{\circ} \mathrm{C}\end{array} \\
6.76\end{array}$} & \multirow{2}{*}{$\begin{array}{c}\begin{array}{c}\text { Salinity } \\
\text { psu }\end{array} \\
34.87\end{array}$} & \multicolumn{3}{|c|}{$\begin{array}{c}{ }^{238} U \\
\operatorname{dpm} \cdot L^{-1}\end{array}$} & \multicolumn{3}{|c|}{$\begin{array}{c}{ }^{234} \mathrm{Th} \\
\mathrm{dpm} \cdot \mathrm{L}^{-1}\end{array}$} & \multirow{2}{*}{$\begin{array}{c}{ }^{234} \mathrm{Th} /{ }^{238} \mathrm{U} \\
0.79\end{array}$} \\
\hline & & & & 2.43 & \pm & 0.05 & 1.92 & \pm & 0.06 & \\
\hline \multirow{16}{*}{$\begin{array}{c}\# 51 \\
59.8^{\circ} \mathrm{N} /-42.0^{\circ} \mathrm{E} \\
18 / 06 / 2014\end{array}$} & 19 & 6.65 & 34.88 & 2.43 & \pm & 0.05 & 1.89 & \pm & 0.05 & 0.78 \\
\hline & 40 & 6.18 & 34.95 & 2.43 & \pm & 0.05 & 2.18 & \pm & 0.07 & 0.90 \\
\hline & 60 & 5.96 & 34.97 & 2.43 & \pm & 0.05 & 2.07 & \pm & 0.06 & 0.85 \\
\hline & 79 & 5.58 & 34.95 & 2.43 & \pm & 0.05 & 2.20 & \pm & 0.06 & 0.91 \\
\hline & 100 & 5.51 & 34.95 & 2.43 & \pm & 0.05 & 2.44 & \pm & 0.07 & 1.00 \\
\hline & 119 & 5.27 & 34.93 & 2.43 & \pm & 0.05 & 2.58 & \pm & 0.07 & 1.06 \\
\hline & 139 & 5.13 & 34.92 & 2.43 & \pm & 0.05 & 2.42 & \pm & 0.07 & 1.00 \\
\hline & 159 & 5.16 & 34.94 & 2.43 & \pm & 0.05 & 2.31 & \pm & 0.06 & 0.95 \\
\hline & 178 & 4.93 & 34.92 & 2.43 & \pm & 0.05 & 2.44 & \pm & 0.07 & 1.01 \\
\hline & 199 & 4.99 & 34.94 & 2.43 & \pm & 0.05 & 2.53 & \pm & 0.07 & 1.04 \\
\hline & 298 & 4.97 & 34.96 & 2.43 & \pm & 0.05 & 2.51 & \pm & 0.07 & 1.03 \\
\hline & 396 & 4.66 & 34.95 & 2.43 & \pm & 0.05 & 2.41 & \pm & 0.07 & 0.99 \\
\hline & 495 & 4.51 & 34.94 & 2.43 & \pm & 0.05 & 2.26 & \pm & 0.06 & 0.93 \\
\hline & 593 & 4.17 & 34.92 & 2.43 & \pm & 0.05 & 2.30 & \pm & 0.06 & 0.94 \\
\hline & 692 & 4.05 & 34.91 & 2.43 & \pm & 0.05 & 2.49 & \pm & 0.07 & 1.02 \\
\hline & 791 & 4.01 & 34.92 & 2.43 & \pm & 0.05 & 2.46 & \pm & 0.07 & 1.01 \\
\hline \multirow{8}{*}{$\begin{array}{c}\# 53 \\
59.9^{\circ} \mathrm{N} /-43.1^{\circ} \mathrm{E} \\
16 / 06 / 2014\end{array}$} & 10 & -0.73 & 31.91 & 2.19 & \pm & 0.05 & 1.75 & \pm & 0.06 & 0.80 \\
\hline & 20 & -1.21 & 32.14 & 2.21 & \pm & 0.05 & 2.08 & \pm & 0.10 & 0.94 \\
\hline & 40 & -1.24 & 32.80 & 2.26 & \pm & 0.05 & 2.02 & \pm & 0.06 & 0.89 \\
\hline & 59 & -1.47 & 33.05 & 2.28 & \pm & 0.05 & 2.20 & \pm & 0.07 & 0.96 \\
\hline & 79 & -1.31 & 33.16 & 2.29 & \pm & 0.05 & 2.21 & \pm & 0.07 & 0.96 \\
\hline & 99 & -0.79 & 33.36 & 2.31 & \pm & 0.05 & 2.08 & \pm & 0.07 & 0.90 \\
\hline & 119 & 0.84 & 33.56 & 2.32 & \pm & 0.05 & 2.03 & \pm & 0.06 & 0.87 \\
\hline & 138 & -0.08 & 33.59 & 2.33 & \pm & 0.05 & 1.91 & \pm & 0.06 & 0.82 \\
\hline \multirow{15}{*}{$\begin{array}{c}\# 64 \\
59.1^{\circ} \mathrm{N} /-46.1^{\circ} \mathrm{E} \\
20 / 06 / 2014\end{array}$} & 9 & 6.55 & 34.80 & 2.42 & \pm & 0.05 & 1.89 & \pm & 0.10 & 0.78 \\
\hline & 20 & 6.04 & 34.85 & 2.42 & \pm & 0.05 & 2.00 & \pm & 0.07 & 0.83 \\
\hline & 40 & 5.93 & 34.87 & 2.43 & \pm & 0.05 & 1.88 & \pm & 0.07 & 0.78 \\
\hline & 79 & 5.37 & 34.95 & 2.43 & \pm & 0.05 & 2.46 & \pm & 0.09 & 1.01 \\
\hline & 99 & 5.35 & 34.96 & 2.43 & \pm & 0.05 & 2.57 & \pm & 0.09 & 1.06 \\
\hline & 139 & 5.17 & 34.96 & 2.43 & \pm & 0.05 & 2.43 & \pm & 0.09 & 1.00 \\
\hline & 159 & 4.92 & 34.94 & 2.43 & \pm & 0.05 & 2.43 & \pm & 0.09 & 1.00 \\
\hline & 197 & 4.78 & 34.93 & 2.43 & \pm & 0.05 & 2.31 & \pm & 0.08 & 0.95 \\
\hline & 297 & 4.51 & 34.93 & 2.43 & \pm & 0.05 & 2.33 & \pm & 0.09 & 0.96 \\
\hline & 396 & 4.35 & 34.93 & 2.43 & \pm & 0.05 & 2.45 & \pm & 0.09 & 1.01 \\
\hline & 494 & 4.10 & 34.91 & 2.43 & \pm & 0.05 & 2.54 & \pm & 0.09 & 1.04 \\
\hline & 594 & 3.97 & 34.90 & 2.43 & \pm & 0.05 & 2.52 & \pm & 0.09 & 1.04 \\
\hline & 692 & 3.88 & 34.89 & 2.43 & \pm & 0.05 & 2.42 & \pm & 0.09 & 1.00 \\
\hline & 792 & 3.71 & 34.88 & 2.43 & \pm & 0.05 & 2.46 & \pm & 0.09 & 1.01 \\
\hline & 890 & 3.64 & 34.87 & 2.43 & \pm & 0.05 & 2.46 & \pm & 0.09 & 1.02 \\
\hline
\end{tabular}




\begin{tabular}{|c|c|c|c|c|c|c|c|c|c|c|}
\hline \multirow[t]{2}{*}{$\begin{array}{c}\text { Station } \\
\# \\
\end{array}$} & \multirow{2}{*}{$\begin{array}{c}\text { Depth } \\
\text { m } \\
11\end{array}$} & \multirow{2}{*}{$\begin{array}{c}\begin{array}{c}\text { Temperature } \\
{ }^{\circ} \mathrm{C}\end{array} \\
6.23\end{array}$} & \multirow{2}{*}{$\begin{array}{c}\text { Salinity } \\
\text { psu }\end{array}$} & \multicolumn{3}{|c|}{$\begin{array}{c}{ }^{238} \mathrm{U} \\
\mathrm{dpm} \cdot \mathrm{L}^{-1}\end{array}$} & \multicolumn{3}{|c|}{$\begin{array}{c}{ }^{234} \mathrm{Th} \\
\mathrm{dpm} \cdot \mathrm{L}^{-1}\end{array}$} & \multirow{2}{*}{$\frac{{ }^{234} \mathrm{Th} /{ }^{238} \mathrm{U}}{0.66}$} \\
\hline & & & & 2.41 & \pm & 0.05 & 1.58 & \pm & 0.05 & \\
\hline \multirow{15}{*}{$\begin{array}{c}\# 69 \\
55.8^{\circ} \mathrm{N} /-48.1^{\circ} \mathrm{E} \\
23 / 06 / 2014\end{array}$} & 20 & 6.15 & 34.61 & 2.41 & \pm & 0.05 & 1.84 & \pm & 0.05 & 0.77 \\
\hline & 59 & 3.73 & 34.77 & 2.42 & \pm & 0.05 & 2.58 & \pm & 0.09 & 1.07 \\
\hline & 80 & 3.92 & 34.83 & 2.42 & \pm & 0.05 & 2.52 & \pm & 0.08 & 1.04 \\
\hline & 99 & 3.83 & 34.82 & 2.42 & \pm & 0.05 & 2.62 & \pm & 0.08 & 1.08 \\
\hline & 119 & 3.95 & 34.85 & 2.42 & \pm & 0.05 & 2.55 & \pm & 0.08 & 1.05 \\
\hline & 139 & 3.91 & 34.86 & 2.42 & \pm & 0.05 & 2.45 & \pm & 0.08 & 1.01 \\
\hline & 159 & 3.83 & 34.86 & 2.42 & \pm & 0.05 & 2.41 & \pm & 0.07 & 0.99 \\
\hline & 199 & 3.58 & 34.84 & 2.42 & \pm & 0.05 & 2.61 & \pm & 0.08 & 1.08 \\
\hline & 298 & 3.54 & 34.85 & 2.42 & \pm & 0.05 & 2.56 & \pm & 0.08 & 1.05 \\
\hline & 496 & 3.51 & 34.85 & 2.42 & \pm & 0.05 & 2.33 & \pm & 0.07 & 0.96 \\
\hline & 595 & 3.48 & 34.85 & 2.42 & \pm & 0.05 & 2.50 & \pm & 0.08 & 1.03 \\
\hline & 693 & 3.47 & 34.85 & 2.42 & \pm & 0.05 & 2.37 & \pm & 0.07 & 0.98 \\
\hline & 792 & 3.48 & 34.85 & 2.42 & \pm & 0.05 & 2.53 & \pm & 0.08 & 1.04 \\
\hline & 890 & 3.48 & 34.85 & 2.42 & \pm & 0.05 & 2.32 & \pm & 0.08 & 0.96 \\
\hline & 990 & 3.46 & 34.85 & 2.42 & \pm & 0.05 & 2.36 & \pm & 0.07 & 0.98 \\
\hline \multirow{15}{*}{$\begin{array}{c}\# 77 \\
53.0^{\circ} \mathrm{N} /-51.1^{\circ} \mathrm{E} \\
26 / 06 / 2014\end{array}$} & 10 & 6.92 & 34.49 & 2.40 & \pm & 0.05 & 1.80 & \pm & 0.07 & 0.75 \\
\hline & 20 & 6.27 & 34.56 & 2.40 & \pm & 0.05 & 1.76 & \pm & 0.07 & 0.73 \\
\hline & 39 & 5.11 & 34.64 & 2.41 & \pm & 0.05 & 2.12 & \pm & 0.08 & 0.88 \\
\hline & 59 & 4.09 & 34.71 & 2.41 & \pm & 0.05 & 2.39 & \pm & 0.10 & 0.99 \\
\hline & 79 & 3.60 & 34.74 & 2.42 & \pm & 0.05 & 2.27 & \pm & 0.07 & 0.94 \\
\hline & 100 & 3.49 & 34.76 & 2.42 & \pm & 0.05 & 2.64 & \pm & 0.08 & 1.09 \\
\hline & 119 & 3.44 & 34.77 & 2.42 & \pm & 0.05 & 2.52 & \pm & 0.08 & 1.04 \\
\hline & 139 & 3.41 & 34.79 & 2.42 & \pm & 0.05 & 2.57 & \pm & 0.08 & 1.06 \\
\hline & 159 & 3.43 & 34.81 & 2.42 & \pm & 0.05 & 2.64 & \pm & 0.08 & 1.09 \\
\hline & 199 & 3.46 & 34.83 & 2.42 & \pm & 0.05 & 2.36 & \pm & 0.08 & 0.97 \\
\hline & 298 & 3.53 & 34.85 & 2.42 & \pm & 0.05 & 2.57 & \pm & 0.09 & 1.06 \\
\hline & 397 & 3.57 & 34.86 & 2.42 & \pm & 0.05 & 2.67 & \pm & 0.09 & 1.10 \\
\hline & 495 & 3.52 & 34.86 & 2.42 & \pm & 0.05 & 2.60 & \pm & 0.09 & 1.07 \\
\hline & 595 & 3.56 & 34.87 & 2.43 & \pm & 0.05 & 2.49 & \pm & 0.08 & 1.03 \\
\hline & 693 & 3.49 & 34.86 & 2.43 & \pm & 0.05 & 2.69 & \pm & 0.08 & 1.11 \\
\hline
\end{tabular}


Table S2: Particulate ${ }^{234} \mathrm{Th}$ activities and POC concentrations in the small (SSF; 1-53 $\mu \mathrm{m}$ ) and large size fraction (LSF;

$>53 \mu \mathrm{m}$ ).

\begin{tabular}{|c|c|c|c|c|c|c|c|c|c|c|c|c|c|}
\hline Station & Depth & \multicolumn{6}{|c|}{$1-53 \mu \mathrm{m}$ (SSF) } & \multicolumn{6}{|c|}{$>53 \mu \mathrm{m}(\mathrm{LSF})$} \\
\hline \# & $\mathrm{m}$ & \multicolumn{3}{|c|}{$\begin{array}{c}{ }^{234} \mathrm{Th} \\
\mathrm{dpm} \mathrm{L}{ }^{-1}\end{array}$} & \multicolumn{3}{|c|}{$\begin{array}{c}\text { POC } \\
\mu \mathrm{mol} \mathrm{L-1}\end{array}$} & \multicolumn{3}{|c|}{$\begin{array}{l}{ }^{234} \mathrm{Th} \\
\mathrm{dpm} \mathrm{L} \mathrm{L}^{-1}\end{array}$} & \multicolumn{3}{|c|}{$\begin{array}{c}\text { POC } \\
\mu \mathrm{mol} \mathrm{L}^{-1}\end{array}$} \\
\hline \multirow{6}{*}{$\begin{array}{c}\# 1 \\
40.3^{\circ} \mathrm{N} \\
-10.0^{\circ} \mathrm{E}\end{array}$} & 30 & 0.163 & \pm & 0.005 & 2.639 & \pm & 0.099 & 0.021 & \pm & 0.001 & 0.651 & \pm & 0.009 \\
\hline & 80 & 0.086 & \pm & 0.003 & 0.208 & \pm & 0.01 & 0.022 & \pm & 0.001 & .357 & \pm & 0.005 \\
\hline & 120 & 0.188 & \pm & 0.006 & 0.567 & \pm & 0.035 & 0.021 & \pm & 0.001 & 0.105 & \pm & 0.005 \\
\hline & 250 & 0.171 & \pm & 0.004 & 0.428 & \pm & 0.019 & 0.038 & \pm & 0.001 & 0.068 & \pm & 0.006 \\
\hline & 550 & 0.123 & \pm & 0.004 & 0.217 & \pm & 0.016 & 0.014 & \pm & 0.000 & 0.023 & \pm & 0.002 \\
\hline & 800 & 0.068 & \pm & 0.003 & 0.035 & \pm & 0.0 & 0.011 & \pm & 0.001 & 0.024 & \pm & 0.005 \\
\hline \multirow{5}{*}{$\begin{array}{c}\# 13 \\
41.4^{\circ} \mathrm{N} \\
-13.9^{\circ} \mathrm{E}\end{array}$} & 30 & 456 & \pm & 0.004 & 2.160 & \pm & 0.0 & 0.028 & \pm & 0.001 & 0.196 & \pm & 0.005 \\
\hline & 80 & 0.529 & \pm & 0.004 & 1.170 & \pm & 0.0 & 0.030 & \pm & 0.001 & 0.080 & \pm & 0.004 \\
\hline & 120 & 0.431 & \pm & 0.004 & & \pm & & & \pm & 0.001 & 0.039 & \pm & 0.005 \\
\hline & 250 & 210 & \pm & 0.002 & 0.366 & \pm & 0. & 020 & \pm & 0.001 & 0.027 & \pm & 0.003 \\
\hline & 450 & 33 & \pm & 0.001 & 0.266 & \pm & & 09 & \pm & 0.000 & .018 & \pm & 0.002 \\
\hline \multirow{6}{*}{$\begin{array}{c}\# 21 \\
46.5^{\circ} \mathrm{N} \\
-19.7^{\circ} \mathrm{E}\end{array}$} & 15 & 80 & \pm & 0.009 & 6.053 & \pm & & & \pm & 0.003 & .747 & \pm & 0.020 \\
\hline & 60 & 0.775 & \pm & 0.017 & 4.064 & \pm & 0.1 & 243 & \pm & 0.005 & 1.201 & \pm & 0.031 \\
\hline & 100 & 0.225 & \pm & 0.005 & 0.545 & \pm & 0.023 & 0.052 & \pm & 0.001 & 0.133 & \pm & 0.007 \\
\hline & 200 & 0.122 & \pm & 0.003 & 0.360 & \pm & 0.021 & 0.038 & \pm & 0.001 & 0.046 & \pm & 0.002 \\
\hline & 450 & 0.114 & \pm & 0.003 & 0.234 & \pm & 0.0 & 0.022 & \pm & 0.000 & 0.023 & \pm & 0.002 \\
\hline & 800 & 108 & \pm & 0.002 & 0.158 & \pm & 0.0 & 14 & \pm & 0.000 & 0.017 & \pm & 0.001 \\
\hline \multirow{4}{*}{$\begin{array}{c}\# 26 \\
50.3^{\circ} \mathrm{N} \\
-22.6^{\circ} \mathrm{E}\end{array}$} & 30 & 279 & \pm & 0.008 & 4.792 & \pm & & 06 & \pm & 0.004 & 2.572 & \pm & 0.017 \\
\hline & 83 & 225 & \pm & 0.005 & 0.848 & \pm & & & \pm & 0.002 & 0.877 & \pm & 0.007 \\
\hline & 153 & 131 & \pm & 0.003 & 0.400 & \pm & 0.0 & 30 & \pm & 0.001 & 0.123 & \pm & 0.003 \\
\hline & 400 & 11 & \pm & 0.005 & 0.240 & \pm & & 12 & \pm & 0.001 & 0.035 & \pm & 0.007 \\
\hline \multirow{6}{*}{$\begin{array}{c}\# 32 \\
55.5^{\circ} \mathrm{N} \\
-26.7^{\circ} \mathrm{E}\end{array}$} & 30 & 09 & \pm & 0.003 & 5.830 & \pm & 0.0 & 45 & \pm & 0.002 & 0.292 & \pm & 0.014 \\
\hline & 60 & 0.359 & \pm & 0.007 & 1.565 & \pm & 0.027 & 027 & \pm & 0.001 & 0.124 & \pm & 0.004 \\
\hline & 100 & 0.224 & \pm & 0.005 & 0.887 & \pm & 0.024 & 0.080 & \pm & 0.002 & 0.338 & \pm & 0.007 \\
\hline & 200 & 0.121 & \pm & 0.004 & 0.467 & \pm & 0.0 & 10 & \pm & 0.000 & 0.046 & \pm & 0.003 \\
\hline & 450 & 078 & \pm & 0.002 & 0.240 & \pm & & & \pm & 0.000 & 0.037 & \pm & 0.002 \\
\hline & 800 & 84 & \pm & 0.002 & 0.203 & \pm & & & \pm & 0.000 & 66 & \pm & 0.002 \\
\hline \multirow{4}{*}{$\begin{array}{c}\# 38 \\
58.8^{\circ} \mathrm{N} \\
-31.3^{\circ} \mathrm{E}\end{array}$} & 20 & 39 & \pm & 0.0 & 2.231 & \pm & & & \pm & & 56 & \pm & \\
\hline & 60 & 90 & \pm & 0.006 & 1.273 & \pm & & & \pm & 0.001 & 0.128 & \pm & 0.004 \\
\hline & 109 & 43 & \pm & 0.003 & 0.621 & \pm & & & \pm & 0.001 & 0.156 & \pm & 0.005 \\
\hline & 396 & & \pm & 0.004 & 0.292 & \pm & & & \pm & 0.000 & 0.030 & \pm & 0.005 \\
\hline \multirow{6}{*}{$\begin{array}{c}\# 44 \\
59.6^{\circ} \mathrm{N} \\
-38.9^{\circ} \mathrm{E}\end{array}$} & 20 & 88 & \pm & 0.020 & 6.736 & \pm & & & \pm & 0.007 & 3.965 & \pm & 0.020 \\
\hline & 40 & 0.474 & \pm & 0.010 & 3.550 & \pm & 0.056 & 0.041 & \pm & 0.001 & 0.133 & \pm & 0.013 \\
\hline & 80 & 1.060 & \pm & 0.028 & 6.736 & \pm & 0.2 & 0.098 & \pm & 0.004 & 0.306 & \pm & 0.046 \\
\hline & 150 & 0.116 & \pm & 0.004 & 0.392 & \pm & & 0.005 & \pm & 0.000 & 0.026 & \pm & 0.009 \\
\hline & 30 & & \pm & 0.003 & 0.334 & \pm & & & \pm & 0.000 & 0.022 & \pm & 0.005 \\
\hline & 500 & & \pm & 0.002 & 0.187 & \pm & & & \pm & 0.000 & 0.016 & \pm & 0.004 \\
\hline \multirow{7}{*}{$\begin{array}{c}\# 51 \\
59.8^{\circ} \mathrm{N} \\
-42.0^{\circ} \mathrm{E}\end{array}$} & 8 & & \pm & 0.007 & & \pm & 0.0 & 0.230 & \pm & 0.005 & 2.664 & \pm & 0.017 \\
\hline & 20 & 58 & \pm & 0.025 & 8.974 & \pm & 0.1 & & & & & & \\
\hline & 60 & 366 & \pm & 0.009 & 2.154 & \pm & 0.036 & 0.148 & \pm & 0.003 & 0.554 & \pm & 0.010 \\
\hline & 70 & 451 & \pm & 0.009 & 2.375 & \pm & 0.034 & & & & & & \\
\hline & 100 & 0.179 & \pm & 0.006 & 0.832 & \pm & 0.032 & 0.052 & \pm & 0.002 & 0.153 & \pm & 0.007 \\
\hline & 15 & 193 & \pm & 0.006 & 0.747 & \pm & & & & & & & \\
\hline & 250 & 0.088 & \pm & 0.003 & 0.328 & \pm & 0.021 & 0.017 & \pm & 0.001 & 0.076 & \pm & 0.005 \\
\hline
\end{tabular}




\begin{tabular}{|c|c|c|c|c|c|c|c|c|c|c|c|c|c|}
\hline \multirow{2}{*}{$\begin{array}{c}\text { Station } \\
\text { \# }\end{array}$} & \multirow{2}{*}{$\begin{array}{c}\text { Depth } \\
\text { m }\end{array}$} & \multicolumn{6}{|c|}{$1-53 \mu \mathrm{m}$ (SSF) } & \multicolumn{6}{|c|}{$>53 \mu \mathrm{m}(\mathrm{LSF})$} \\
\hline & & \multicolumn{3}{|c|}{$\begin{array}{c}{ }^{234} \mathrm{Th} \\
\mathrm{dpm} \mathrm{L} \mathrm{L}^{-1}\end{array}$} & \multicolumn{3}{|c|}{$\begin{array}{c}\text { POC } \\
\mu \mathrm{mol} \mathrm{L}^{-1}\end{array}$} & \multicolumn{3}{|c|}{ 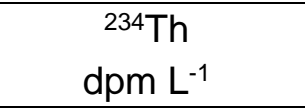 } & \multicolumn{3}{|c|}{$\begin{array}{c}\text { POC } \\
\mu \mathrm{mol} \mathrm{L}{ }^{-1}\end{array}$} \\
\hline & 30 & 024 & \pm & 0.001 & 0.418 & \pm & 0.009 & 026 & \pm & 0.001 & .373 & \pm & 0.002 \\
\hline$\#$ & 60 & 0.174 & \pm & 0.007 & 1.726 & \pm & 0.0 & .015 & \pm & 0.001 & 0.154 & \pm & 0.012 \\
\hline 59. & 100 & 0.162 & \pm & 0.004 & 1.968 & \pm & 0.028 & 0.030 & \pm & 0.001 & 0.267 & \pm & 0.006 \\
\hline \multirow[t]{3}{*}{$-46.1^{\circ} \mathrm{E}$} & 150 & 0.118 & \pm & 0.005 & 0.189 & \pm & 0.046 & 0.035 & \pm & 0.002 & 0.208 & \pm & 0.007 \\
\hline & 400 & 0.113 & \pm & 0.003 & 0.309 & \pm & & 0.010 & \pm & 0.000 & 0.054 & \pm & 0.004 \\
\hline & 8 & 0.206 & \pm & 0.007 & 4.130 & \pm & 0.0 & 0.070 & \pm & 0.002 & 1.077 & \pm & 0.013 \\
\hline & & & \pm & 0.0 & 1.9 & \pm & & & \pm & 0.001 & 0.172 & \pm & 0. \\
\hline $55.8^{\circ}$ & 100 & & \pm & 0.004 & 1.6 & \pm & & & \pm & 0.001 & 0.299 & \pm & 0.007 \\
\hline \multirow[t]{3}{*}{$-48.1^{\circ} \mathrm{E}$} & 150 & 97 & \pm & 0.004 & 0.895 & \pm & & 0.010 & \pm & 0.001 & 0.083 & \pm & 0.009 \\
\hline & 410 & & \pm & 0.002 & 0.313 & \pm & & 0.006 & \pm & 0.000 & 0.075 & \pm & 0.005 \\
\hline & 10 & 13 & \pm & 0.011 & 11.182 & \pm & & 0.206 & \pm & 0.006 & 3.039 & \pm & 0.021 \\
\hline & 50 & 0.447 & \pm & 0.012 & 5.488 & \pm & 0.067 & 0.057 & \pm & 0.002 & 0.425 & \pm & 0.016 \\
\hline $53.0^{\circ} \mathrm{N}$ & 80 & 0.150 & \pm & 0.004 & 2.347 & \pm & 0.028 & 0.039 & \pm & 0.001 & 0.339 & \pm & 0.007 \\
\hline \multirow{2}{*}{$-51.1^{\circ} \mathrm{E}$} & 200 & 0.100 & \pm & 0.004 & 0.533 & \pm & 0.040 & 0.009 & \pm & 0.000 & 0.081 & \pm & 0.008 \\
\hline & 460 & 0.069 & \pm & 0.003 & 0.167 & \pm & 0.017 & 0.011 & \pm & 0.000 & 0.060 & \pm & 0.004 \\
\hline
\end{tabular}


a)

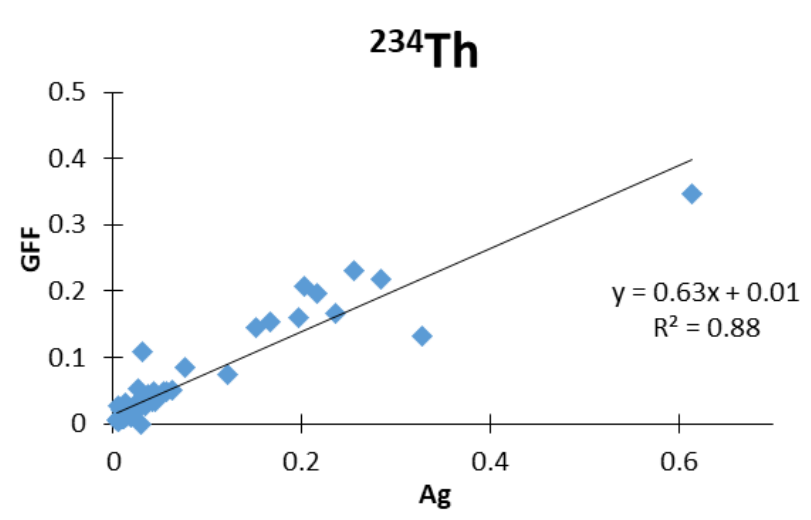

b)

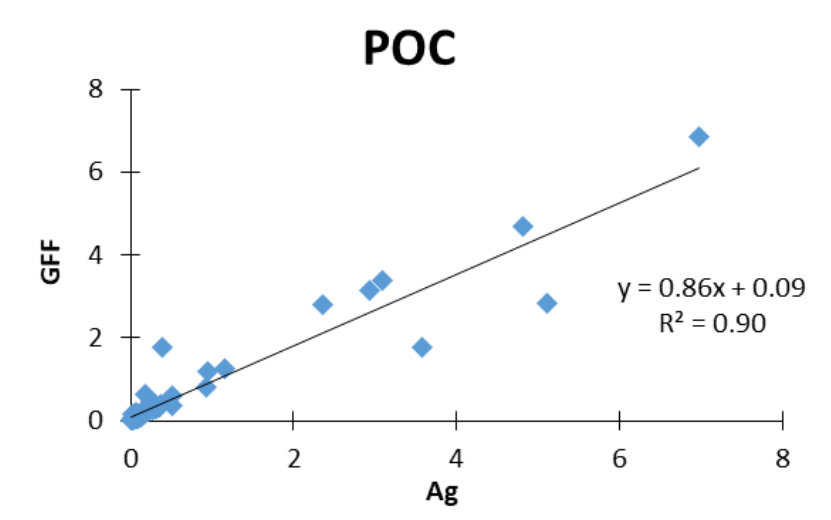

Figure S1: Comparison between a) ${ }^{234}$ Th activities and b) POC concentrations measured on GF/F and silver (Ag) filters. 


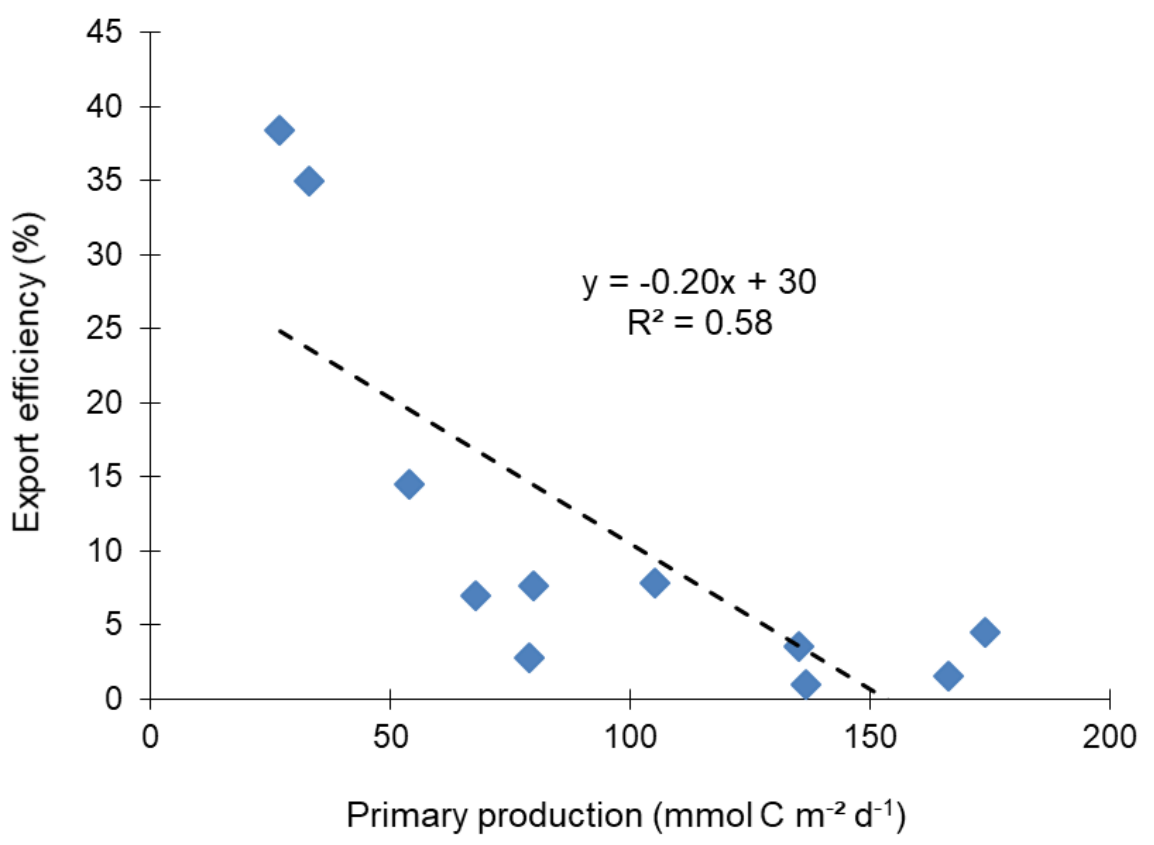

Figure S2: Export efficiency (in \%) as a function of primary production (mmol $\left.\mathrm{C} \mathrm{m}^{-2} \mathbf{d}^{-1}\right)$. 